\title{
Safety and Relative Dose Intensity of Dose-dense Doxorubicin and Cyclophosphamide Followed by Dose-dense Paclitaxel
}

\author{
MEIKO NISHIMURA ${ }^{1}$, TAKUMA ONOE $^{1}$, HIDEKI SAKAI $^{1}$, MINORI ARASE $^{2}$, \\ SAYURI WATANABE ${ }^{2}$, MISAO SOYAMA ${ }^{3}$, KAZUKI HASHIMOTO $^{3}$, MAYUKO MIKI $^{3}$, \\ KAORI TANE $^{3}$, KOICHI HIROKAGA ${ }^{3}$, SHINTARO TAKAO ${ }^{3}$ and KOJI MATSUMOTO ${ }^{1}$ \\ ${ }^{1}$ Department of Medical Oncology, Hyogo Cancer Center, Akashi, Japan; \\ ${ }^{2}$ Department of Pharmacy, Hyogo Cancer Center, Akashi, Japan; \\ ${ }^{3}$ Department of Breast Surgery, Hyogo Cancer Center, Akashi, Japan
}

\begin{abstract}
Background/Aim: Dose-dense doxorubicin and cyclophosphamide (ddAC) followed by dose-dense paclitaxel $(d d P)$ (ddAC-P) has improved disease-free survival of patients with breast cancer. The aim of this study was to evaluate the safety and relative dose intensity (RDI) of ddAC-P administered together with pegfilgrastim. Patients and Methods: Between May 2015 and Aug 2017, 44 patients were retrospectively reviewed; they were administered 4 cycles of ddAC, followed by 4 cycles of ddP. Pegfilgrastim (3.6 mg) was administered in every cycle. Results: The mean RDIs for $d d A C-P$, $d d A C$, and $d d P$ were $95.0 \%, 94.5 \%$, and $93.3 \%$, respectively. The prevalence of high RDIs ( $\geq 85 \%)$ for $d d A C-P$, $d d A C$, and $d d P$ was $90.9 \%, 84.1 \%$, and $88.6 \%$, respectively. Seven of the 10 patients with low RDIs experienced grade 1 or 2 fever. Conclusion: DdAC-P administered together with pegfilgrastim (3.6 $\mathrm{mg}$ ) appears to be feasible and maintains RDI in most of Japanese patients with breast cancer. Rapid evaluation and proper management of fever may prevent low RDI.
\end{abstract}

Compared to conventional chemotherapy, dose-dense doxorubicin and cyclophosphamide (ddAC) followed by dosedense paclitaxel (ddP) (ddAC-P) serves as a perioperative chemotherapy regimen that improves disease-free survival of patients with breast cancer (1). This treatment requires preventive granulocyte-colony stimulating factor (G-CSF) as the primary prophylaxis. G-CSF had no indication for patients

Correspondence to: Koji Matsumoto, Department of Medical Oncology, Hyogo Cancer Center, 13-70, Kitaoji-cho, Akashi-City, Hyogo, 673-8558, Japan. Tel: +81 789291151, Fax: +81 789292387, e-mail: kojmatsu2@hyogo-cc.jp

Key Words: Dose-dense doxorubicin and cyclophosphamide, dosedense paclitaxel, breast cancer, pegfilgrastim, perioperative chemotherapy. with breast cancer as primary prophylaxis, and pegfilgrastim was not approved in Japan, until November 2014. At that time, pegfilgrastim was approved in Japan at a dose of $3.6 \mathrm{mg}$, according to data obtained from registration trials, included pharmacokinetics and dose-identification study in the country (2). The Pharmaceuticals and Medical Devices Agency indicated pegfilgrastim for patients with breast cancer as primary prophylaxis based on the results of the registration trials that evaluated docetaxel and cyclophosphamide or docetaxel, doxorubicin, and cyclophosphamide, but not ddAC$P(3,4)$. This lack of safety data regarding the combined use of ddAC-P and pegfilgrastim at a dose of $3.6 \mathrm{mg}$ has resulted in the inclusion of a cautionary sentence in the package inserts of pegfilgrastim in Japan.

To elucidate the feasibility of ddAC-P regimen in Japanese patients, a retrospective study was conducted to analyze the safety aspects and relative dose intensity (RDI) in patients with breast cancer receiving $\mathrm{ddAC}-\mathrm{P}$ along with pegfilgrastim $(3.6 \mathrm{mg})$. To the best of our knowledge, this is the first report on the feasibility of ddAC-P supported by pegfilgrastim at a dose of $3.6 \mathrm{mg}$ in Japanese patients with breast cancer.

\section{Patients and Methods}

Patients. Between May 2015 and Aug 2017, 44 patients with breast cancer who were administered 4 cycles of ddAC-P, doxorubicin $\left(60 \mathrm{mg} / \mathrm{m}^{2}\right)$, and cyclophosphamide $\left(600 \mathrm{mg} / \mathrm{m}^{2}\right)$ every 14 days, followed by 4 cycles of paclitaxel $\left(175 \mathrm{mg} / \mathrm{m}^{2}\right)$ every 14 days were retrospectively reviewed. Pegfilgrastim $(3.6 \mathrm{mg})$ was subcutaneously administered on day 3 of every ddAC-P cycle.

This study was approved by the Institutional Review Boards of Hyogo Cancer Center, and all the patients provided written informed consent.

Assessment. Adverse events were independently assessed by pharmacists using the Common Terminology Criteria for Adverse Events (version 4.0). In the neoadjuvant setting, the anti-tumor efficacy was evaluated by magnetic resonance imaging and 
ultrasound after chemotherapy on the basis of the Response Evaluation Criteria in Solid Tumors (version 1.1).

We assessed the RDI of ddAC-P, ddAC, and ddP. RDI of $\geq 85 \%$ was defined as "high RDI," whereas that of $<85 \%$ was defined as "low RDI." The prevalence of high RDI and the factors responsible for low RDI were also investigated.

\section{Results}

Patient characteristics. Patient characteristics are summarized in Table I. The median age of the patients was 51.5 years (age range $=34-69$ years). All patients belonged to Eastern Cooperative Oncology Group performance status (ECOG PS) 0 or 1. Stage IIA, IIB, and IIIA accounted for $77.3 \%$ of cases; $75 \%$ of cases were ER/PgR ${ }^{+}, \mathrm{HER}^{-}$, and $25 \%$ were $\mathrm{ER} / \mathrm{PgR}^{-}, \mathrm{HER}^{-}$. The neoadjuvant setting was represented by $43.2 \%$ of the cases.

Hospitalization for monitoring. Treatment-related death was not observed. To be on the safe side, patients with adverse events were proactively hospitalized for effective monitoring. As a result of this policy, the recovery of five patients with adverse events was monitored by maintaining them as inpatients. Three patients with fever (grade 2 fever on day 15 of the 4 th cycle of ddAC, grade 1 fever on day 15 of the 1 st cycle of ddP, and grade 1 fever on day 16 of the 2nd cycle of ddP), one patient with dizziness (grade 2 on day 7 of the 4th cycle of ddAC), and one patient with pneumonitis (grade 3 on day 18 of the 4 th cycle of ddAC) were hospitalized for approximately 1 week. Four of the five patients rapidly recovered after being treated only with hydration. None of them received intensive care, such as vasopressor therapy or respiratory management. One patient with pneumonitis received oxygen for 3 days and was discharged after 6 days of hospitalization.

Adverse events. Adverse events are summarized in Table II. The common $(\geq 50 \%)$ non-hematological toxicities were peripheral neuropathy $(90.5 \%)$ and arthritis/myalgia (79\%). Most of these were grade 1 or 2 , with only two patients experiencing grade 3 peripheral neuropathy. None of the patients experienced grade 3 or 4 arthritis/myalgia. Eight (18.2\%) patients had fever, and all were either grade 1 or 2 . The most common hematological toxicity was lymphopenia $(52.3 \%)$. The patients neither experienced febrile neutropenia $(\mathrm{FN})$ nor did they require blood transfusion. One patient $(2.3 \%)$ on ddP developed grade 2 pneumonitis; we could not exclude Pneumocystis jiroveci pneumonia (PCP) owing to the presence of a ground-glass pattern on computed tomography (CT) images; hence, a treatment course of trimethoprim/sulfamethoxazole (TMP/SMX) $(15 \mathrm{mg} / \mathrm{kg}$ ) was initiated. However, serum $\beta$-D-glucan levels did not increase, and the patient recovered 7 days later in the absence of steroid treatment. One patient $(2.3 \%)$ on ddAC developed grade 3 pneumonitis. We suspected PCP or interstitial lung
Table I. Patient characteristics

\begin{tabular}{lc}
\hline Characteristic & $\mathrm{n}(\%)$ \\
\hline Age - years & \\
$\quad$ Median (range) & $51.5(34-69)$ \\
ECOG PS & \\
0 & $38(86.4)$ \\
1 & $6(13.6)$ \\
Stage & \\
IA, IB, & $5(11.4)$ \\
IIA, IIB, IIIA & $34(77.3)$ \\
IIIB, IIIC, & $4(9.0)$ \\
IV & $1(2.3)$ \\
Subtype & \\
ER/PgR ${ }^{+}$, HER2 \\
ER/PgR ${ }^{-}$, HER2 & \\
Treatment setting & $33(75.0)$ \\
Neoadjuvant & $11(25.0)$ \\
Adjuvant & \\
Surgery & $19(43.2)$ \\
Lumpectomy & $25(56.8)$ \\
Mastectomy & $11(25.0)$ \\
\hline
\end{tabular}

ECOG PS: Eastern Cooperative Oncology Group performance status.

Table II. Adverse events (AE)

\begin{tabular}{lcccc}
\hline AEs & \multicolumn{5}{c}{$\mathrm{n}(\%)$} \\
\cline { 2 - 5 } & Grade 1 & Grade 2 & Grade 3 & Grade 4 \\
\hline Fever & $7(15.9)$ & $1(2.3)$ & 0 & 0 \\
Febrile neutropenia & 0 & 0 & 0 & 0 \\
Pneumonitis & 0 & $1(2.3)$ & $1(2.3)$ & 0 \\
Lung infection & 0 & $1(2.3)$ & 0 & 0 \\
Nausea & - & - & $1(2.3)$ & 0 \\
Peripheral sensory neuropathy & $22(50.0)$ & $16(36.0)$ & $2(4.5)$ & 0 \\
Arthritis/Myalgia & $15(34.0)$ & $20(45.0)$ & 0 & 0 \\
Lymphopenia & - & - & $22(50.0)$ & $1(2.3)$ \\
Neutropenia & - & - & $2(4.5)$ & $1(2.3)$ \\
\hline
\end{tabular}

-: Not assessed.

disease (ILD) because of the ground-glass pattern observed on $\mathrm{CT}$, and the patient recovered following a course of TMP/SMX without steroids. Bronchoscopy was not performed, which precluded a definitive diagnosis. Nevertheless, serum $\beta$-D-glucan levels did not increase, suggesting that ILD was more probable than PCP.

Treatment discontinuation, dose delay, and dose reduction. $\mathrm{ddP}$ was discontinued in cycle 1 in one patient $(2.3 \%)$, because of strong anxiety after the development of grade 2 pneumonitis. Treatment delay occurred in $34.1 \%$ of ddACtreated patients and in $31.8 \%$ of ddP-treated patients. The 


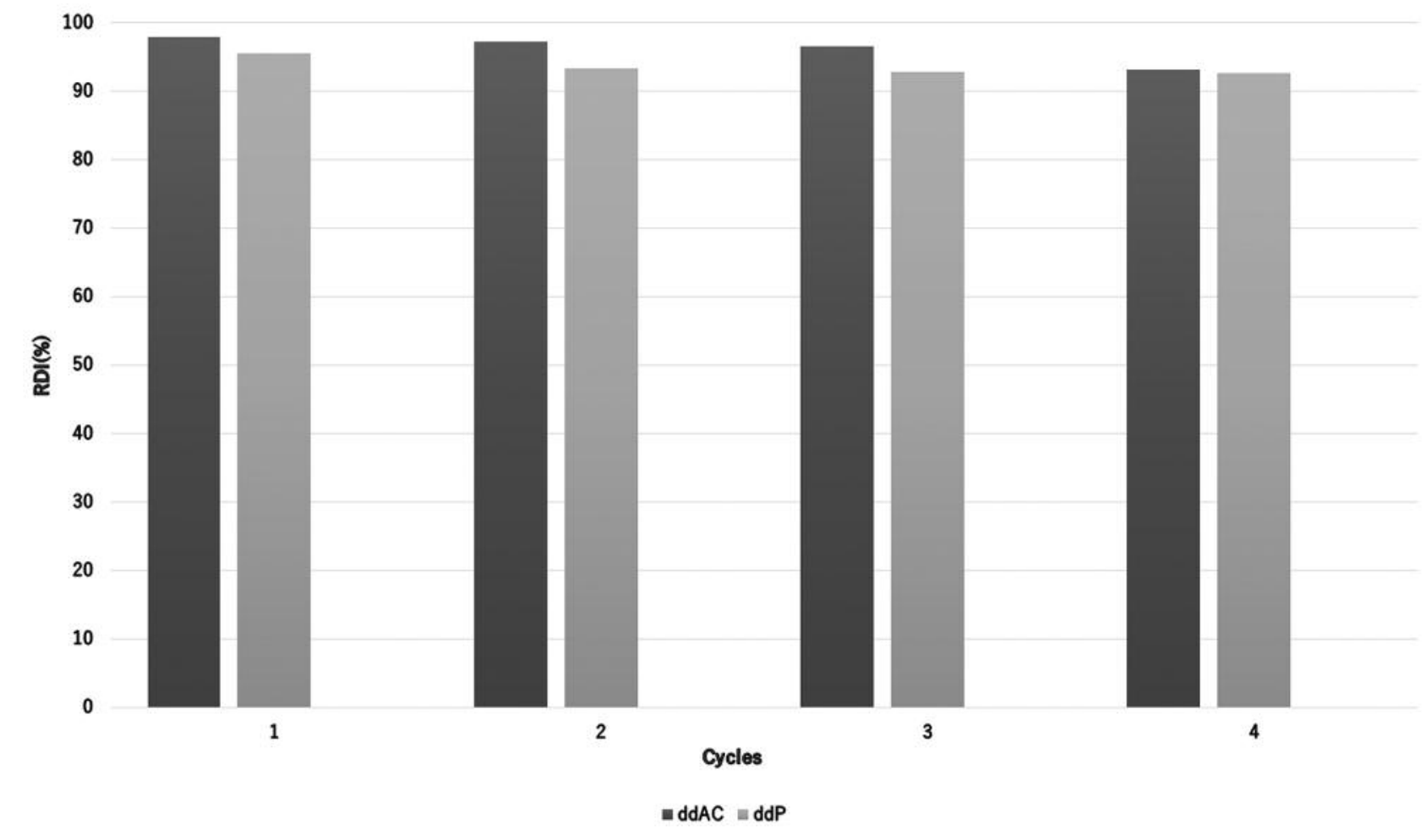

Figure 1. The mean percent relative dose intensity (RDI) per treatment cycle. Black bars represent ddAC and gray bars represent ddP. The mean RDI of cycles 1 - 4 were $98 \%, 97.2 \%, 96.6 \%$, and $93.2 \%$ for $d d A C$, and $95.6 \%, 93.3 \%, 92.9 \%$, and $92.6 \%$ for ddP, respectively.

major reasons for delaying ddAC therapy were holiday $(33.3 \%, 5 / 15)$, grade $1 / 2$ fever in cycles 1,3 , and $4(33.3 \%$, $5 / 15)$, and grade $1 / 2$ upper respiratory infection $(13.3 \%$, $2 / 15)$ in cycles 1 and 2 . The prime factors for delaying ddP treatment were holiday $(35.7 \%, 5 / 14)$ and grade 1 fever in cycles 1 and $2(28.6 \%, 4 / 14)$.

Although no dose reduction occurred in the ddAC-treated patients, it was required in $22.7 \%(\mathrm{n}=10)$ of the ddP-treated patients. The major contributing factors were grade $2 / 3$ peripheral sensory neuropathy $(80 \%)$ during cycle $2(1 / 8)$, cycle $3(3 / 8)$, and cycle $4(4 / 8)$. Additionally, dose reduction was required in one patient for grade 2 myalgia.

$R D I$. The mean percent of RDIs per treatment cycle are shown in Figure 1. The mean values for ddAC-P, ddAC, and ddP were $95.0 \%, 94.5 \%$, and $93.3 \%$, respectively. The percentage of prevalence of high RDI $(\geq 85 \%)$ for ddAC-P, ddAC, and ddP was $90.9 \%, 84.1 \%$, and $88.6 \%$, respectively. Low RDI was observed in 10 patients owing to grade 1 or 2 fever (7/10; Table III).

The risk factors that may account for the low RDI were also investigated. The proportion of elderly patients $(>65$ years) with low RDI $v s$. high RDI for ddAC were $28.6 \%$ (2/7) vs. $8.1 \%$ (3/37) (odds ratio 4.7, $p=0.15$; Fisher's exact
Table III. Adverse events (AE) associated with low RDI

\begin{tabular}{ll}
\hline AEs & $\mathrm{n}(\%)$ \\
\hline Fever (grade 1, 2) & $7(70.0)$ \\
Pneumonitis (grade 3) & $1(10.0)$ \\
Other $^{\dagger}$ & $2(20.0)$ \\
\hline
\end{tabular}

$\dagger$ Other AEs were herpes zoster and symptoms that resembled encephalitis.

test). Although the difference was not significant, the trend suggests that elderly patients may be at risk of low RDI. Eight patients developed grade 1 or 2 fever. Seven of the eight patients (87.5\%) with fever suffered from low RDI.

Treatment efficacy. Among the 19 patients who received ddAC-P as neoadjuvant chemotherapy, the clinical complete response (cCR) rate and clinical partial response (cPR) rates were $15.8 \%(n=3)$ and $68.4 \%(n=13)$, respectively. In the neoadjuvant setting, the rate of lumpectomy was $21.1 \%$ $(n=4)$.

The median duration of follow-up was 17.8 months, and all patients survived without recurrence. 


\section{Discussion}

This is the first study that evaluated the safety aspects and the RDI of ddAC-P supported by pegfilgrastim $(3.6 \mathrm{mg})$. No mortality or FN occurred in the study patients; Intensive care unit (ICU) admission was also not required. This safety profile (no treatment-related death, no ICU admission, and no FN) is comparable with that reported in previous studies. Previous studies regarding ddAC-P (or ddEC-P) include CALGB9741, Gruppo Italiano Mammla 2 (GIM2), and SWOG0221 (1, 5, 6). All these investigations employed pegfilgrastim $(6.0 \mathrm{mg})$ or daily filgrastim. Mizuno et al. and Morita et al. have studied ddAC (or ddEC) using pegfilgrastim $(6.0 \mathrm{mg})$; ddP was not included in both the studies $(7,8)$.

The mean RDI was $>90 \%$ for ddAC-P, ddAC, and ddP; this result is consistent with the RDI reported in the GIM2 study (5). Almost $90 \%$ patients achieved high RDI, which is also comparable with that reported in previous studies on ddAC or ddEC $(5,7,8)$.

Furthermore, our data suggest that the primary reason for low RDI was grade 1 or 2 fever, and no other risk factors were detected. Grade $1 / 2$ and grade $3 / 4$ fever were observed in $18 \%$ and $0 \%$ of patients in our study; this result is not higher than that obtained in the previous GIM 2 study $(26 \%$ and $<1 \%)$. There were no other risk factors, such as age, and complications in patients experienced fever. PCP has been reported in patients with breast cancer receiving ddAC; hence, careful examinations and observations were ensured in case of fever as it may cause low RDI $(9,10)$.

In a previous study of a ddAC-P regimen (CALGB9741), blood transfusion and hospitalization for FN were observed in $3 \%$ and $2 \%$ of cases, respectively (1). Treatment-related death was observed in $0.4 \%$ of patients on ddAC and in $0.3 \%$ of those on ddP in the SWOG S0221 trial (6). However, FN and treatment-related deaths were not observed in our study, and blood transfusions were not required. These toxicity data indicated that low-dose pegfilgrastim $(3.6 \mathrm{mg})$ is not associated with increased toxicity in Japanese patients.

Limitations of our study include the small sample size and its retrospective nature. Therefore, a phase 2 study on ddAC$\mathrm{P}$ has already been initiated to provide more information (West Japan Oncology Group; WJOG 9016B).

In conclusion, ddAC-P plus pegfilgrastim $(3.6 \mathrm{mg})$ is a feasible therapeutic strategy that maintains RDI in Japanese patients with breast cancer. Fever was the most common adverse event associated with low RDI. Management of this complication is therefore critical in maintaining the RDI.

\section{Conflicts of Interest}

The Authors have no conflicts of interest.

\section{Authors' Contributions}

M. Nishimura, K. Matsumoto, and S. Takao wrote the manuscript. $\mathrm{M}$. Arase and S. Watanabe assessed the adverse events.

\section{Acknowledgements}

The Authors would like to thank all the participants in this study, including patients, caregivers, physicians, and medical workers.

\section{References}

1 Citron ML, Berry DA, Cirrincione C, Hudis C, Winer EP, Gradishar WJ, Davidson NE, Martino S, Livingston R, Ingle JN, Perez EA, Carpenter J, Hurd D, Holland JF, Smith BL, Sartor CI, Leung EH, Abrams J, Schilsky RL, Muss HB and Norton L: Randomized trial of dose-dense versus conventionally scheduled and sequential versus concurrent combination chemotherapy as postoperative adjuvant treatment of node-positive primary breast cancer: first report of Intergroup Trial C9741/Cancer and Leukemia Group B Trial 9741. J Clin Oncol 21(8): 1431-1439, 2003. PMID: 12668651. DOI: 10.1200/JCO.2003.09.081

2 Miyazaki Y, Kubo K, Murayama T, Usui N, Urabe A, Tamura K and Hotta T: A multicenter, double-blind, randomized Phase III study comparing KRN125 with filgrastim in lymphoma. Jpn J Clin Hematol 54: 1064, 2013. DOI: 10.11406/rinketsu.54.1043

3 Masuda N, Tokuda Y, Nakamura S, Shimazaki R, Ito Y and Tamura K: Dose response of pegfilgrastim in Japanese breast cancer patients receiving six cycles of docetaxel, doxorubicin, and cyclophosphamide therapy: a randomized controlled trial. Support Care Cancer 23(10): 2891-2898, 2015. PMID: 25733 000. DOI: $10.1007 / \mathrm{s} 00520-015-2654-4$

4 Kosaka Y RY, Masuda N, Takano T, Saeki T, Nakamura S, Shimazaki R, Ito Y, Tokuda Y and Tamura K: Phase III placebocontrolled, double-blind, randomized trial of pegfilgrastim to reduce the risk of febrile neutropenia in breast cancer patients receiving docetaxel/cyclophosphamide chemotherapy. Support Care Cancer 23(4): 1137-1143, 2015. PMID: 25576433. DOI: 10.1007/s00520-014-2597-1

5 Del Mastro L, De Placido S, Bruzzi P, De Laurentiis M, Boni C, Cavazzini G, Durando A, Turletti A, Nistico C, Valle E, Garrone O, Puglisi F, Montemurro F, Barni S, Ardizzoni A, Gamucci T, Colantuoni G, Giuliano M, Gravina A, Papaldo P, Bighin C, bisagni G, Forestieri V and Cognetti F: Fluorouracil and dosedense chemotherapy in adjuvant treatment of patients with earlystage breast cancer: an open-label, $2 \times 2$ factorial, randomised phase 3 trial. Lancet 385(9980): 1863-1872, 2015. PMID: 25740286. DOI: 10.1016/s0140-6736(14)62048-1

6 Budd GT, Barlow EW, Moore HC, Hobday TJ, Stewart JA, Isaacs C, Salim M, Cho JK, Rinn KJ, Albain KS, Chew HK, Burton GV, Moore TD, Srkalovic G, McGregor BA, Flaherty LE, Livingston RB, Lew DL, Gralow JR and Hortobagyi GN: SWOG S0221: a phase III trial comparing chemotherapy schedules in high-risk early-stage breast cancer. J Clin Oncol 33(1): 58-64, 2015. PMID: 25422488. DOI 10.1200/JCO.2014. 56.3296

7 Mizuno Y, Fuchikami H, Takeda N, Iwai M and Sato K: Efficacy of reduced dose of pegfilgrastim in Japanese breast cancer 
patients receiving dose-dense doxorubicin and cyclophosphamide therapy. Jpn J Clin Oncol 47(1): 12-17, 2017. PMID: 28122890. DOI: $10.1093 /$ jjco/hyw 152

8 Morita S, Kikumori T, Tsunoda N, Inaishi T, Adachi Y, Ota A, Shibata M, Matsuoka A, Nakanishi K, Takeuchi D, Mizutani T, Shimokata T, Hayashi H, Maeda O and Ando Y: Feasibility of dose-dense epirubicin and cyclophosphamide with subcutaneous pegfilgrastim $3.6 \mathrm{mg}$ support: a single-center prospective study in Japan. Int J Clin Oncol 23(1): 195-200, 2018. PMID: 28791509. DOI: 10.1007/s10147-017-1177-z

9 Waks AG, Tolaney SM, Galar A, Arnaout A, Porter JB, Marty FM, Winer EP, Hammond SP and Baden LR: Pneumocystis jiroveci pneumonia (PCP) in patients receiving neoadjuvant and adjuvant anthracycline-based chemotherapy for breast cancer: incidence and risk factors. Breast Cancer Res Treat 154(2): 359367, 2015. PMID: 26420402. DOI: 10.1007/s10549-015-3573-2 10 Tolaney SM, Partridge AH, Sheib RG, Burstein HJ and Winer EP: Pneumocystis carinii pneumonia during dose-dense chemotherapy for breast cancer. J Clin Oncol 24(33): 5330-5331, 2006. PMID: 17114668. DOI: 10.1200/JCO.2006.08.1083

Received May 16, 2019

Revised June 11, 2019

Accepted June 12, 2019 